\title{
Notes de lecture et généalogie des idées
}

Le discours apologétique de Jeufroy et la pensée maistrienne

\section{Taro Nakajima}

\section{(2) OpenEdition}

\section{Journals}

Édition électronique

URL : http://journals.openedition.org/aes/574

DOI : $10.4000 /$ aes. 574

ISSN : 2258-093X

Éditeur

Laboratoire LISAA

Référence électronique

Taro Nakajima, " Notes de lecture et généalogie des idées », Arts et Savoirs [En ligne], 1 | 2012, mis en ligne le 15 février 2012, consulté le 22 avril 2019. URL : http://journals.openedition.org/aes/574 ; DOI : 10.4000/aes.574

Ce document a été généré automatiquement le 22 avril 2019.

Centre de recherche LISAA (Littératures SAvoirs et Arts) 


\title{
Notes de lecture et généalogie des idées
}

\author{
Le discours apologétique de Jeufroy et la pensée maistrienne
}

\author{
Taro Nakajima
}

1 Théoricien de la contre-révolution, apologiste d'une philosophie de l'autorité contre les Lumières ${ }^{1}$, Joseph de Maistre est l'un des écrivains catholiques qui nourrissent le discours apologétique de l'abbé Jeufroy dans le neuvième chapitre (religion) de Bouvard et Pécuchet. Comme le montre la liste bibliographique établie par Stéphanie Dord-Crouslé, on connaît la place prépondérante voire privilégiée et pléthorique ${ }^{2}$, que le penseur savoyard occupe dans les notes de lecture prises par Flaubert pour le neuvième chapitre et pour le second volume du roman encyclopédique.

2 Ce qui nous intéresse ici, c'est l'inscription des notes de lecture dans le texte du roman final plutôt que la question de leur transfert dans le second volume ${ }^{3}$, ou la voix de l'écrivain dans la mise en texte des savoirs ${ }^{4}$ dont la critique a déjà montré l'importance. Notre champ d'étude se limite à l'utilisation des notes de lecture dans le discours du prêtre : il s'agit d'un ensemble complexe d'idées reçues religieuses, constitué à partir d'ouvrages de piété de différentes époques et de divers genres, dont les composantes restent encore à être éclaircies ${ }^{5}$. Montage hétéroclite, l'argumentation de Jeufroy subit pourtant une orientation idéologique particulière selon le scénario sur le chapitre de la religion qui explique le désaccord entre l'ecclésiastique et les deux bonshommes : « [ceuxci] discutent avec le curé et les raisons du curé leur enlèvent leur embryon de croyance. Ce qui les détache, c'est le côté de Maistre, les soi-disant preuves historiques et les raisons politiques $»^{6}$.

3 Il est d'ailleurs tout à fait naturel de supposer que le personnage de Jeufroy a été conçu comme un prêtre «maistrien » si l'on se rappelle que Flaubert prétend avoir tout lu de «l'odieux Joseph de Maistre $»^{7}$ : l'écrivain a pris des notes non seulement sur des ouvrages majeurs du philosophe, Considérations sur la France, Essai sur le principe générateur ..., Du Pape, Les Soirées de Saint-Pétersbourg, Éclaircissement sur les sacrifices, mais encore sur des recueils des lettres publiés bien plus tard après la disparition de l'auteur, tels que 
Lettres et opuscules inédits et Correspondance diplomatique ${ }^{8}$. Il est donc incontestable que le romancier a entrepris une lecture exhaustive de ce «hideux, [l']exécrable "Monsieur de Maistre" »".

4 Or, en comparant les notes de lecture et le texte du roman, on s'aperçoit que des nombreuses notes prises sur de Maistre (et qui auraient ensuite nourri le second volume) il reste très peu de traces dans les débats théologiques du neuvième chapitre, et cela lors même que les arguments de Jeufroy, discutant avec les deux héros sceptiques, incarnent par excellence la pensée et la rhétorique maistriennes. Comment penser ce déséquilibre entre l'abondance des notes de lecture et la quasi-absence de leurs traces dans le roman? Quelles sont les références des arguments du curé s'ils ne sont pas tirés de de Maistre ? En examinant l'utilisation d'autres écrivains catholiques postérieurs à celui-ci dans les discussions religieuses du roman, on s'interrogera sur une généalogie des idées autour de ce penseur savoyard, l'une des grandes figures du catholicisme intransigeant du XIX ${ }^{\mathrm{e}}$ siècle.

\section{Le péché originel ou le dogme de la réversibilité}

5 Dans le neuvième chapitre, on trouve une première trace de la pensée maistrienne dans la discussion sur le péché originel. Après avoir lu un sommaire d'exégèse moderne envoyé par son ami Barberou, Bouvard a des doutes sur le dogme du péché originel en se demandant comment Dieu doit punir l'homme s'il l'a créé peccable. À son objection rationaliste insistant sur l'injustice de la sentence divine («ce dogme bouleverse mes notions de justice !»), Jeufroy répond :

- «Que voulez-vous » disait le curé « c'est une de ces vérités dont tout le monde est

d'accord sans qu'on puisse en fournir de preuves; - et nous-mêmes, nous faisons rejaillir sur les enfants les crimes de leurs pères. Ainsi les mœurs et les lois justifient ce décret de la Providence, que l'on retrouve dans la nature. $»^{10}$

6 Il s'agit d'un dogme fondamental sur lequel repose la vision maistrienne du péché originel, le dogme de la réversibilité, que de Maistre évoquait dans les Considérations sur la France (1797) et développera plus tard dans Les Soirées de Saint-Pétersbourg, composées en 1809 et publiées en 1821. La punition des fils pour les crimes de leurs pères est sans doute un mystère inconcevable au premier abord. Mais cela ne s'explique-t-il pas par le dogme de la réversibilité selon lequel les douleurs de l'innocent profitent au coupable, dogme établi dans tout l'univers? Ce dogme n'appartient pas uniquement au christianisme mais il existe en tout temps et en tout lieu, et les pratiques sacrificielles chez les anciens prouvent, bien que sous des formes altérées, la croyance en une malédiction héréditaire difficile à expliquer par le raisonnement.

7 Or, les notes de lecture de Flaubert montrent que l'opinion de Jeufroy n'est pas ici, contrairement à ce que l'on attendait, tirée d'ouvrages de Maistre mais d'un autre ouvrage apologétique, Le catholicisme présenté dans l'ensemble de ses preuves (1859) de Fernand Baguenault de Puchesse, publiciste et conseiller municipal, associé à l'activité religieuse et politique de l'évêque d'Orléans :

Les hommes imitent Dieu quand ils font rejaillir sur les enfants les fautes des pères

- quand ils font suivre de générations en générations la honte \& le déshonneur quand ils semblent en un mot avoir tout établi dans leurs mœurs, tout combiné dans leurs institutions pour justifier ce décret de Dieu qu'on retrouve aussi de toute part dans les lois physiques de la nature. ${ }^{11}$ 
On y retrouve les énoncés constitutifs de la seconde moitié de l'opinion de Jeufroy. Flaubert raccourcit le texte original de quelques lignes en en modifiant légèrement certains mots («honte» mis pour «ruine»), mais il résume bien l'idée principale de l'auteur. Baguenault divise son livre en six parties où il explique tour à tour différentes «preuves» du catholicisme, naturelles, historiques, scientifiques, dogmatiques, morales et philosophiques, pour montrer l'accord parfait de la science et de la religion. Il s'agit ici des preuves « dogmatiques » (4 partie). Voici les pages prises en note par Flaubert:

Avec le sang s'est transmise la tache, comme se seraient communiqués le mérite et la gloire. Tel est l'arrêt de Dieu : arrêt sans doute mystérieux et inexplicable, mais que les hommes, avec une justice infiniment moindre et une connaissance infiniment moins parfaite, imitent tous les jours, quand ils font rejaillir sur les enfants les fautes et les punitions du père, quand, en dépouillant et abaissant l'un, ils dépouillent et abaissent en même temps les autres, quand ils font suivre ainsi de générations en générations la ruine et le déshonneur, quand ils semblent, en un mot, avoir tout établi dans leurs mœurs, tout combiné dans leurs institutions pour justifier ce décret de Dieu qu'on retrouve aussi de toute part dans les lois physiques de la nature. ${ }^{12}$

9 Si le mot « Providence » n'apparaît pas ici, l'idée de Baguenault selon laquelle les hommes imitent quotidiennement l'arrêt de Dieu renvoie à la théorie de la réversibilité chez Maistre, «un des esprits les plus élevés de notre siècle ${ }^{13}$ selon l'auteur. On sait que l'affirmation du péché originel « continué $»^{14}$ chez de Maistre, peu orthodoxe, s'apparente à une hérésie en ce qu'elle souligne l'aspect répétitif du péché d'Adam que chaque individu imite, selon lui, de sa propre volonté : «Le péché originel, qui explique tout et sans lequel on n'explique rien, se répète malheureusement à chaque instant de la durée, quoique d'une manière secondaire. $»^{15}$ C'est là, comme le rappelle $\mathrm{A}$. Compagnon, que la doctrine maistrienne du péché originel rejoint curieusement la théologie protestante de son temps telle que la représente Schleiermacher ${ }^{16}$.

10 Maistre est l'un des grands penseurs les plus cités dans Le catholicisme présenté de Baguenault qui réunit diverses preuves de la religion sous la forme d'« un résumé court et suffisamment complet $»^{17}$. Ainsi, dans la section sur les sacrifices et le «besoin d'une expiation ", la démonstration repose en grande partie sur la pensée maistrienne (l'auteur cite Les Soirées et Éclaircissement sur les sacrifices). Même s'il ne précise pas ses références, on trouve parfois dans ses arguments une rhétorique traditionaliste qui évoque le penseur savoisien: "Ainsi donc, aussi haut qu'on remonte dans l'histoire des peuples, aussi loin qu'on s'écarte des régions qui furent le berceau du genre humain, toujours et partout on retrouve, plus ou moins pures, plus ou moins intactes, les grandes croyances à Dieu, à son pouvoir créateur, à sa providence ${ }^{18}$.

11 De plus, Baguenault cite non seulement les ouvrages de Maistre mais utilise les mêmes sources que celui-ci, telles que les Recherches asiatiques et les travaux de William Jones et de Soame Jenyns, sans préciser qu'il les avait empruntées à l'auteur des Soirées. Pour parer à tout reproche de plagiat, il explique la pratique citationnelle de son ouvrage constitué de nombreux emprunts faits aux grands écrivains : «Nous avons parfois, et malgré nous, omis de citer quelques-uns de ces emprunts qui se trouvaient confondus dans notre texte et étaient devenus méconnaissables pour nous-mêmes $»^{19}$. L'appropriation similaire des savoirs religieux précédents caractérise le discours de Jeufroy, ensemble cohérent mais d'origine hétérogène, où s'entassent comme des strates des fragments empruntés à divers apologistes, dont il est difficile de démêler les provenances, comme pour le cas du péché originel. Ainsi, d'après les notes de lecture 
prises par Flaubert ( $f^{\circ} 292$ ), la première moitié de la citation de Jeufroy (« c'est une de ces vérités dont tout le monde est d'accord sans qu'on puisse en fournir de preuves ») qui évoque la formule concessive maistrienne («sans doute, mais... »") provient en réalité d'un autre ouvrage apologétique célèbre, Le Génie du christianisme de Chateaubriand. Il s'agit d'une présence un peu inattendue dans ce contexte puisque l'auteur des Essais sur les révolutions n'a pas toujours partagé les idées des penseurs contre-révolutionnaires comme de Maistre ${ }^{21}$.

Dans Les Soirées, de Maistre n'emploie pas le terme "rejaillir», mais il explique la transmission héréditaire de la faute originelle par la métaphore de "maladie originelle». Or, l'une des "partialités $~^{22}$ remarquables de ses notes de lecture, c'est que Flaubert a sauté ce passage du deuxième entretien, tout à fait essentiel pour comprendre les notions maistriennes de péché originel et de réversibilité. Au chevalier, jeune interlocuteur, se demandant si l'on doit souffrir pour les crimes d'un autre commis il y a plus d'un siècle, le comte, le porte-parole de l'auteur, répond :

Tout être qui a la faculté de se propager ne saurait produire qu'un être semblable à lui. La règle ne souffre pas d'exception : elle est écrite sur toutes les parties de l'univers. Si donc un être est dégradé, sa postérité ne sera plus semblable à l'état primitif de cet être, mais bien à l'état où il a été ravalé par une cause quelconque. [...] La maladie aiguë n'est pas transmissible; mais celle qui vicie les humeurs devient maladie originelle, et peut gâter toute une race. ${ }^{23}$

Si Maistre ne fait pas de distinction entre les maladies, physiques ou morales, c'est qu'« elles sont toutes des châtiments ». Certes Flaubert n'en a rien retenu dans ses notes, mais dans le roman, cette théorie de la ressemblance justifiant la transmission entre pères et fils peut motiver la correction des deux enfants vicieux par Jeufroy qui les trouve d'autant plus dégradés qu'ils sont nés d'un forçat. Dans la défense du péché originel par le curé s'inscrit donc la doctrine peu orthodoxe de de Maistre qui donne une " actualité » à la faute d'Adam ${ }^{24}$, qu'il tente d'expliquer toujours par l'universalité des traditions.

\section{La loi de substitution}

Dans le neuvième chapitre, on trouve une autre trace de la pensée maistrienne dans la discussion sur la rédemption suivie d'une scène de chasse aux lapins. Avant de donner une fessée à Victor qui s'avère vicieux, on l'amène dans la forêt pour lui montrer une chasse sanglante où les animaux innocents sont abattus de coups de fusil et la blouse de l'enfant souillée de sang:

La vue du sang répugnait à Bouvard. Il n'admettait pas qu'on en pût verser.

M. Jeufroy reprit : - «Les circonstances quelquefois l'exigent. Si ce n'est pas le coupable qui donne le sien, il faut celui d'un autre, - vérité que nous enseigne la Rédemption. » (347)

L'argument de Jeufroy renvoie à la théorie maistrienne de la substitution, indissociable du dogme de la réversibilité que l'on a vu plus haut. La question du sacrifice sanglant et de sa vertu expiatoire, le thème central de l'Éclaircissement sur les sacrifices, apparait déjà au neuvième entretien des Soirées : «Les hommes, affirme le comte, n'ont jamais douté que l'innocent ne pût satisfaire pour le crime; et ils ont cru de plus qu'il y avait dans le sang une force expiatrice; de manière que la vie, qui est le sang, pouvait racheter une autre vie $»^{25}$. Une autre particularité surprenante des notes de Flaubert, c'est qu'il n'a pratiquement pas pris de notes pour ce neuvième entretien, capital dans Les Soirées, dont les réflexions prolongent celles des Considérations sur la France en même temps qu'elles 
préparent celles de l'Éclaircissement, composé en 1810 et publié avec Les Soirées. Or, le romancier semble avoir bien compris l'importance du dogme de la substitution dans la métaphysique maistrienne, comme en témoignent ses notes récapitulatives sur l' Éclaircissement qui se trouvent à la fin des notes de lecture sur Les Soirées : «La théorie de De M. est celle-ci : L'homme est déchu (même dans les idées de l'antiquité.) la vie est dans le sang. - l'innocent paye $\mathrm{p}^{\mathrm{r}}$. le coupable. Donc on donne la vie de l'innocent $\mathrm{p}^{\mathrm{r}}$. racheter celle du coupable - et c'est très bien ${ }^{26}$.

Il s'agit d'une relecture de la théorie maistrienne des sacrifices, car on sait que Flaubert a lu ce traité des sacrifices lorsqu'il préparait son roman sur Carthage ${ }^{27}$. Dans l' Éclaircissement, Maistre s'interroge sur la signification du sacrifice sanglant par une étude comparative des pratiques religieuses. Si tous les peuples de l'antiquité ont cherché dans les effusions de sang un moyen d'apaiser la colère des dieux, c'est qu'ils auraient compris aussi bien la vertu expiatoire du sang ${ }^{28}$, utile à l'homme et à la société, que la loi de substitution fondée sur le dogme de la réversibilité selon lequel l'innocent peut prendre la place du coupable: «On croyait (comme on a cru, comme on croira toujours) que l'innocent pouvait payer pour le coupable; d'où l'on concluait que la vie étant coupable, une vie moins précieuse pouvait être offerte et acceptée pour une autre $»^{29}$. Ce passage du premier chapitre peut être l'une des sources du résumé de Flaubert sur la théorie maistrienne, où l'on entend la voix auctoriale sous la forme d'une mention ironique ( - et c'est très bien »). Dans l'épisode de la chasse « éducative », le baron de Mahurot, le futur gendre du comte de Faverges (grand admirateur de Maistre, celui-ci revendique le bras de fer dans l'éducation des enfants), aurait choisi comme victime les lapins, "une vie moins précieuse ", qu'il sacrifie pour une autre vie qu'est ici l'enfant coupable de vol, pour lui inculquer vainement le sens du sacrifice sanglant :

M. de Mahurot [...] prit un fusil dans l'antichambre et appela Victor, resté au milieu

de la cour, la tête basse :

- « Suis-moi ! » dit le baron.

Comme la route pour aller chez le garde détournait peu de Chavignolles, M. Jeufroy,

Bouvard et Pécuchet l'accompagnèrent.

À cent pas du château, il les pria de ne plus parler, tant qu'il longerait le bois. [...]

Des lapins sortirent de leurs terrains, et broutaient le gazon

Un coup de feu partit, un deuxième, un autre, - et les lapins sautaient, déboulaient.

Victor se jetait dessus pour les saisir, et haletait trempé de sueur.

- «Tu arranges bien tes nippes » dit le baron. - Sa blouse en loques avait du sang.

(347)

17 Comme Flaubert l'a relevé dans ses notes, Maistre souligne «l'identité du sang et de la vie $»^{30}$, et c'est seulement quand on fait couler le sang des victimes que celles-ci, animales ou humaines, se transforment en un substitut qui peut racheter la vie du coupable. Dans le roman, c'est donc l'idée maistrienne qui semble légitimer un petit carnage d'animaux dont le sang fait des taches sur le vêtement du garçon («Sa blouse en loques avait du sang ») comme si elles rappelaient que celui-ci ne pourrait pas échapper à la malédiction du sang paternel.

Alors que les autres religions ont abusé du dogme de la réversibilité jusqu'à offrir des sacrifices humains, le christianisme a rectifié l'idée universelle de la « rédemption par le sang». Ainsi, Maistre soutient dans le dernier chapitre de l'Éclaircissement que la rédemption du Christ a révélé "l'efficacité merveilleuse du sacrifice volontaire de l'innocence $»^{31}$ et qu'elle est sans cesse prolongée dans le pain et le vin eucharistiques. Lorsque Jeufroy, à la suite de la discussion sur la rédemption, évoque le corps 
perpétuellement sacrifié de Jésus dans l'eucharistie ${ }^{32}$, son argument reprend sous une forme très condensée la conclusion de l'Éclaircissement où Maistre confirme la force salvatrice du sang et la loi de substitution dont la mort du Fils de Dieu n'est que l'exemple le plus illustre.

Contrairement au Sottisier où l'écrivain catholique "a l'honneur d'être le plus cité »"33, on constate très peu d'usage des notes de Flaubert dans le chapitre de la religion (ce qui n'empêche pas Jeufroy d'être un prêtre maistrien). L'exemple de l'Éclaircissement dont on trouve des traces suffisamment visibles dans les arguments du personnage est donc un des rares cas de la mise en œuvre des notes de lecture prises sur les ouvrages du grand apologiste.

\section{« Toujours et partout » : I'immutabilité du dogme}

Flaubert met en scène sous la forme d'un dialogue comique des querelles religieuses entre deux camps idéologiquement opposés, l'un rationaliste et anticlérical, et l'autre clérical et apologétique. Alors que Pécuchet met en doute l'unité permanente du dogme catholique en étudiant l'histoire des dogmes et les contradictions des récits bibliques, Jeufroy s'efforce de la défendre en énumérant plusieurs " preuves ». Selon la thèse traditionaliste de Maistre, les religions de l'Antiquité qu'il considère toutes comme "fausses", préfiguraient cependant, sous des apparences plus ou moins corrompues, les grandes vérités que la religion chrétienne dévoilera plus tard pleinement ; autrement dit, celle-ci « recueille en elle, sous une forme épurée et corrigée, la tradition universelle dont elle extrait et révèle le fonds commun de vérité ॥ $^{34}$.

Face à la mythologie comparée de Pécuchet montrant les origines "plurielles » du christianisme qui ne se ramènent pas à une source unique comme le prétend Jeufroy, celui-ci « ne voulait que le christianisme pût avoir humainement la moindre raison d'être, bien qu'il en vît chez tous les peuples, des prodromes ou des déformations » $(B P, 343)$. Le comte des Soirées refuse lui aussi la raison d'être «humaine " au christianisme pour défendre l'immutabilité du dogme : «Si le christianisme était humain, son enseignement varierait avec les opinions humaines; mais comme il part de l'être immuable, il est immuable comme lui $»^{35}$. Mais c'est notamment chez le sénateur au septième entretien que l'on retrouve une argumentation très proche de celle de Jeufroy: "les fausses religions ont toujours professé la même croyance: car l'erreur, en tournant le dos à sa rivale, ne cesse néanmoins d'en répéter tous les actes et toutes les doctrines qu'elle altère suivant ses forces, c'est-à-dire de manière que le type ne peut jamais être méconnu, ni l'image prise pour lui. ${ }^{36}$ Ici, le terme "erreur » renvoie aux religions autres que le christianisme (on se souvient que Hilarion, le diable de la Tentation de 1874, utilisait le même mot pour montrer à saint Antoine que le martyre n'est pas l'apanage du christianisme ${ }^{37}$ ). Les rapports établis par Maistre entre la vraie religion (le type) et ses variations ou mauvaises copies (l'image) qui en imitent tous les aspects sous des formes erronées peuvent donc nourrir indirectement l'idée de Jeufroy selon laquelle les religions de l'Antiquité constituent des signes avant-coureurs de l'avènement du christianisme. La formule généralisatrice "toujours et partout " récurrente sous la plume de Joseph de Maistre est révélatrice d'une vision anhistorique et innéiste qui refuse de voir toute historicité dans le dogme catholique pour en montrer la vérité immuable, vision parfois réductrice, dont on trouve une trace dans l'assertion de Jeufroy : 
[...] Il retrouvait la Vierge dans les Gaules, l'annonce d'un rédempteur en Chine, la Trinité partout, la croix sur le bonnet du grand lama, en Égypte au poing des dieux ; - et même il fit voir une gravure, représentant un nilomètre, lequel était un phallus suivant Pécuchet. (334) proviennent pas de Joseph de Maistre mais d'un ouvrage d'Adrien Péladan, publiciste légitimiste, Preuves éclatantes de la Révélation par l'histoire universelle ou Monuments et témoignages païens, juifs et de tous les peuples, confirmateurs de la Bible et du christianisme (1878). Le romancier a pris quatre pages de notes ( $f^{\circ} 202 \mathrm{r}^{\circ}$ à $\left.203 \mathrm{v}^{\circ}\right)$ sur la quatrième édition revue et augmentée, tandis que les trois premières ont été publiées sous un autre titre : Histoire de Jésus-Christ d'après la science ( $1^{\text {re }}$ édition, $1865 ; 2^{\mathrm{e}}$ édition, 1866). Comme le montre sa préface à la deuxième édition, cet ouvrage apologétique avait une tâche militante, c'est-à-dire « le renversement complet et sans réplique du système des athées contemporains, sans en excepter le nouveau et insipide roman de M. Renan, Les Apôtres $»^{38}$ . On voit donc l'actualité de l'ouvrage dont l'un des buts principaux fut la réfutation complète du livre de Renan qui venait de paraitre alors.

Péladan oppose à la science moderne orgueilleuse la "vraie science », celle qu « marche d'accord avec la révélation » et dont la lumière interroge l'histoire de la bonne foi. Cherchant des preuves évidentes de la religion dans les monuments et les annales du monde entier, il s'inscrit clairement dans une généalogie de la pensée maistrienne: «Toujours et partout, à notre appel, le monde païen, la synagogue, le mahométisme, les annales de tous les âges, ont justifié la Bible et l'Évangile ${ }^{39}$. La méthode traditionaliste se confirme dans le premier chapitre où l'auteur explique l'idée générale de l'ouvrage, son intention d'accabler les sceptiques sous «les témoignages unanimes des peuples, voix universelle qui parle sur mille rivages, sous toutes les latitudes, dans la succession des siècles $»^{40}$.

Parmi les éléments constituant le discours de Jeufroy, voici ceux qui sont tirés des notes de Flaubert: la sainte Vierge des Gaulois, l'annonce du rédempteur chez les Chinois ( $f^{\circ}$ $202 \mathrm{r}^{\circ}$ ), la Trinité chez tous les peuples, la croix sur le bonnet du grand Lama, la croix comme "phallus» $\left(f^{\circ} 202 v^{\circ}\right)$. Quant aux autres qui ne sont pas pris en note, ils se retrouvent aussi dans l'ouvrage de Péladan (que Flaubert aurait relu après la prise de notes), la croix surmontée d'une anse placée à la main des dieux égyptiens ${ }^{41}$, la croix comme représentation du nilomètre ${ }^{42}$. Adrien Péladan lui-même se réfère à un ouvrage de Creuzer, traduit en français (adapté et complété) par J.-D. Guigniaut, Religions de l'antiquité , présentant une « diversité extrême d'opinions » sur le vrai sens de la figure de la croix ${ }^{43}$. Flaubert a noté au f $202 \mathrm{v}^{\circ}$ des noms de savants qui y ont vu l'image d'un phallus, opinion dénoncée par Péladan comme un « vrai délire d'imagination ». La querelle comique entre Jeufroy et Pécuchet met donc en scène les controverses sur la signification de ce symbole dont rend compte l'ouvrage de Creuzer-Guignaut. Le romancier se serait bien amusé en opposant à la prétendue universalité du signe de la croix une objection insolite de Pécuchet empruntée à l'ouvrage du mythologue allemand - Creuzer - qui l'avait accompagné depuis la première Tentation de saint Antoine (1849).

\section{Intertextualité et généalogie des idées}

Dans sa lutte contre l'exégèse moderne qui sape les bases de la religion, l'ouvrage de Péladan permet donc à Jeufroy de contre-attaquer en lui fournissant des "preuves 
éclatantes » de la révélation, des témoignages de tous les peuples « confirmateurs » de la Bible et du christianisme, comme le rappelle son sous-titre. Mais Jeufroy ne pouvant vérifier lui-même la véracité de ses sources, ne fait que souligner le bien-fondé de leur certitude comme lorsqu'il riposte "par le témoignage des sibylles "dont le fond est véritable" » (334), détail encore tiré de Péladan ${ }^{44}$. La faiblesse du prêtre tient à ce qu'il ne parvient à fournir ses preuves soi-disant authentiques qu'indirectement, par l'intermédiaire d'un autre ou d'un imprimé : «M. Jeufroy consultait secrètement son ami Pruneau, qui lui cherchait des preuves dans les auteurs. Une lutte d'érudition s'engagea ; et fouetté par l'amour-propre Pécuchet devint transcendant, mythologue.» (334) Derrière l'image de Pruneau, collègue du curé, se dessine celle de l'auteur des Preuves éclatantes cherchant lui aussi des preuves « dans les auteurs » faisant autorité, l'auteur des Soirées en particulier.

La pensée maistrienne qui pénètre tout l'ouvrage de Péladan est particulièrement remarquable dans le chapitre sur les sacrifices fondé sur le dogme de la réversibilité ou celui de la substitution que l'on a vu plus haut. Voici les notes prises par Flaubert sur ce chapitre $\left(f^{\circ} 202 v^{\circ}\right)$ :

Les sacrifices Les talmudistes décident que les péchés ne peuvent être effacés que par le sang.

Le dogme du salut par le sang se retrouve partout, il brave le temps \& l'espace

(De Maistre)

«L'homme poussa même ce dogme jusqu'à la démence, en immolant des victimes humaines » 235. - Eh bien ? \& la mort de JC. Est-elle un sacrifice?

«les Druides (dit César) estimaient que le supplice des coupables était qq chose de

fort agréable

à la divinité »

les femmes indiennes.

On peut distinguer cinq énoncés différents associés à "sacrifices ": le premier sur les talmudistes provient d'un ouvrage de Pierre-Daniel Huet cité par Péladan; le deuxième est une citation de Joseph de Maistre comme le précise Flaubert; le troisième (suivi d'ailleurs de son commentaire malveillant, comparant la Passion du Christ aux sacrifices humains de l'Antiquité) est de Péladan lui-même ; le quatrième sur les druides dérive de De Bello Gallico de César; le dernier sur la femme indienne renvoie à deux sources, les Recherches asiatiques de William Jones et la Gazette de France. Or, tous ces éléments se retrouvent dans les notes prises à partir du seul ouvrage de Joseph de Maistre, Éclaircissement sur les sacrifices, auquel Adrien Péladan emprunte de nombreux éléments : dans Preuves éclatantes, il ne fait pas un résumé à proprement parler, il refond le texte original et cite des ouvrages utilisés dans les notes de l'Éclaircissement, juxtaposant ces éléments au texte même de Maistre sans avouer que l'ensemble de ces éléments empruntés à Joseph de Maistre ou aux livres cités par Joseph de Maistre proviennent tous d'un même ouvrage.

Pierre-Sébastien Laurentie, l'un des publicistes lus par Flaubert, a-t-il remarqué cette pratique surprenante de collage inavoué dans un article qui fait l'éloge de Péladan " continuateur et l'illustrateur de Joseph de Maistre $»^{45}$ ? Le travail du plagiaire innocent qui n'hésite pas à remanier librement le texte original du grand apologiste (comme c'est le cas chez Baguenault, mais de manière plus modeste ${ }^{46}$ ) fait preuve d'une appropriation respectueuse mais clandestine de la pensée maistrienne par l'auteur des Preuves éclatantes. Cet ouvrage, qui procède comme l'auteur des Soirées ${ }^{47}$, alimente les «soi-disant preuves historiques » évoquées dans le scénario pour déterminer la position idéologique du curé. 
Ainsi, on se rend compte que, dans les moments apparemment les plus «maistriens ", Flaubert n'utilise pas les notes de lecture prises sur de Maistre mais d'autres éléments empruntés à deux propagateurs de sa pensée, Baguenault de Puchesse et Péladan, dont on a vu les traces dans les discussions sur le dogme du péché originel et l'unanimité des témoignages des peuples. Alors qu'ils ont une même tendance idéologique, un point commun rapproche encore ces deux ouvrages : ils ont été approuvés par un ou plusieurs prélats. La présence de ces certificats qui témoigne d'un monde très hiérarchisé aurait intéressé le romancier qui a décrit dans Madame Bovary un curé de village qui ne cesse de recourir à l'autorité de l'Église pour cacher sa sottise ou son incompétence. Dans Bouvard et Pécuchet, l'abbé Jeufroy s'appuie d'ailleurs sur l'infaillibilité de l'Église (on songe encore à l'auteur de $\mathrm{Du} \mathrm{Pape}^{48}$ ) lorsqu'il est embarrassé. Le catholicisme présenté de Baguenault est approuvé par Mgr Dupanloup, et l'Histoire de Jésus-Christ d'après la science de Péladan accumule dans sa couverture le nom de plusieurs évêques en tête desquels Mgr de Bonald, ce qui indique à quel point l'autorité ecclésiastique était soucieuse de fortifier la foi contre les erreurs modernes par les travaux des apologistes laïques.

Or, lors de sa quatrième édition qui s'intitulera Preuves éclatantes, ouvrage lu par Flaubert, une autre approbation, certainement la plus importante, s'ajoutera à ces lettres d'éminents évêques: un bref apostolique de Pie IX. Ce rescrit du pape ${ }^{49}$, s'adressant amicalement à l'auteur ( « cher notre Fils »), se propose « d'honorer avec joie des marques de notre bienveillance Pontificale, et d'élever par des titres éminents les hommes qui [...] s'efforcent de glorifier et de venger la cause sacrée criminellement attaquée en nos temps $»^{50}$. La lettre est scellée en cire rouge, de l'anneau du Pêcheur, c'est-à-dire «du cachet où saint Pierre est représenté en pêcheur, et qui doit être apposé en présence du pape» (Littré). On pourrait relever d'autres différences entre les deux éditions de Péladan, les reflets de la Commune de Paris ${ }^{51}$, la réprobation des ouvrages de Renan plus atténuée dans la dernière, mais ce qui donne à la version de 1878 un statut prestigieux que n'avait pas celle de 1866, c'est l'ajout de la lettre du pape en tête du livre, remplaçant la plupart des lettres précédentes et attestant que le souverain pontife approuve personnellement l'ouvrage. Le curé de Chavignolles citant des preuves puisées dans le livre de Péladan se réfère donc implicitement à l'autorité pontificale. Dans Madame Bovary , Bournisien se soumettait aussi à l'autorité ecclésiastique ${ }^{52}$. Mais dans le neuvième chapitre de Bouvard, ce n'est pas uniquement Jeufroy qui cherche des preuves authentiques dans les écrits des auteurs catholiques, car son adversaire Pécuchet se réfère lui aussi à un manuel d'exégèse moderne d'un normalien, pour y puiser des connaissances qui lui permettent de critiquer efficacement la religion. Leur «lutte d'érudition » n'est donc qu'une " lutte de citations » dont la bêtise consiste à croire et à respecter aveuglément « ce qui est imprimé » ${ }^{53}$.

\section{Le catholicisme intransigeant}

31 D'une certaine manière, le pape Pie IX et Félix Dupanloup s'inscrivent eux-mêmes dans une généalogie de la pensée maistrienne en approuvant solennellement les travaux des apologistes laïques. Mgr Dupanloup en félicitant Baguenault de Puchesse souhaitait que les hommes du monde se consacrent à la défense de la foi et « viennent, à l'exemple de $\mathrm{M}$. de Bonald, de M. de Maistre, de M. Nicolas, fortifier les rangs des auxiliaires laïques de l'Église, au dix-neuvième siècle ${ }^{54}$. Le théoricien de la contre-révolution demeure vénéré comme un modèle du discours apologétique dans la seconde moitié du siècle. 
La réfutation de la philosophie voltairienne par l'auteur des Soirées est renouvelée dans la lutte menée par l'Église contre la montée du rationalisme moderne, où les ennemis principaux ne sont plus les philosophes des Lumières mais les savants, historiens et philologues. Monseigneur Dupanloup dénonce les travaux récents de Renan, Maury, Taine et Littré dans son fameux Avertissement à la jeunesse et aux pères de famille, écrit à la suite de la publication de la Vie de Jésus par Renan. En prenant des notes sur De la Haute éducation intellectuelle de ce prélat, Flaubert a recopié ceci : «J'en sais (des femmes) qui se sont cru permis de lire la Vie de Jésus, ce roman sacrilège \& ridicule, le plus répugnant ouvrage que je connaisse $»^{55}$. Quant au pape Pie IX, il est connu pour avoir établi dans l'encyclique Quanta cura un catalogue de propositions jugées inacceptables sous le titre de Syllabus errorum, sur lequel Monseigneur Dupanloup écrit une brochure explicative pour apaiser les conflits entre les ultramontains et les catholiques libéraux ${ }^{56}$.

Dans le roman, lorsque Jeufroy perçoit l'évolution historique dont témoigne le discours antireligieux de Pécuchet ("L'impiété railleuse du XVIII ${ }^{\mathrm{e}}$ siècle, il l'eût tolérée; mais la critique moderne avec sa politesse, l'exaspérait » [343]), son constat renvoie ainsi à un combat des catholiques militants des années 1860 contre la libre pensée telle que l'incarne à leurs yeux l'auteur de l'Histoire des origines du christianisme, dont on trouve bien des traces sous la plume de Péladan : «Voltaire attaquait le christianisme par le sarcasme, par la négation à force ouverte, par le mensonge carrément prononcé. M. Renan procède autrement; suppôt du socialisme, [...] il a reçu la mission de saper l'Église par des dissertations doucereuses. Ce trompeur niera bien la divinité de Jésus-Christ, mais il affectera d'entourer le Sauveur d'une auréole divine ou quasi divine $»^{57}$. L'opinion de Jeufroy est donc typique de l'apologétique de la seconde moitié du siècle qui voit une nouvelle forme de critique religieuse remplacer l'ironie voltairienne du siècle précédent.

Ce que nous avons considéré sous le nom de pensée maistrienne - défense traditionaliste ${ }^{58}$ des dogmes immuables, prétendues preuves historiques, dénonciation virulente de la révolution et du rationalisme, insistance sur l'authenticité des livres sacrés, dogme de la réversibilité - a des traits communs avec un courant de la pensée religieuse et politique $\mathrm{du} \mathrm{XIX}^{\mathrm{e}}$ siècle : le catholicisme nommé «intransigeant " par les historiens. Selon Jean Marie Mayeur, celui-ci «se fonde sur un refus total de la société née de la Renaissance, de la Réforme et de la Révolution, dominée par l'individualisme et le rationalisme, la sécularisation de l'État, des sciences, et de la pensée ${ }^{59}$, et les représentants les plus illustres en sont «J. de Maistre, Bonald, le premier Lamennais, au temps de la Restauration, Veuillot, Blanc de Saint-Bonnet, Donoso Cortès, après la crise de $1848 »^{60}$. Le refus de tout compromis avec la modernité peut d'ailleurs se lire dans la dernière proposition du Syllabus de Monseigneur Dupanloup selon laquelle «le pontife romain peut et doit se réconcilier et transiger avec le progrès, avec le libéralisme et la civilisation moderne $»^{61}$, proposition désavouée par le pape.

Ce courant intransigeant qui unit différents milieux catholiques dans un même combat fournit à Flaubert une vulgate, ce qui peut expliquer la quasi-absence de traces des ouvrages de de Maistre sur lesquels le romancier a pourtant pris des notes de lecture abondantes. Quand Flaubert a conçu le personnage de Jeufroy comme un curé maistrien (« le côté de Maistre »), ce qu'il voulait représenter ce n'est certainement pas la théorie de Joseph de Maistre dans son exactitude (il n'a pas pris en note les pages capitales des Soirées), mais la manière dont celle-ci se propage inlassablement dans le discours apologétique militant de la seconde moitié du XIX ${ }^{\mathrm{e}}$ siècle, sous des formes variées : livres de vulgarisation, abrégés, sommaires ${ }^{62}$ dont la pratique citationnelle est parfois proche $d u$ 
plagiat comme on l'a vu. C'est peut-être une des raisons pour lesquelles il s'est servi de ses notes prises sur les deux continuateurs du grand apologiste dans les débats du neuvième chapitre où les arguments du curé semblent incarner l'esprit maistrien.

Parmi les notables de Chavignolles, on trouve une autre figure incarnant cette filiation intransigeante : le comte de Faverges, royaliste légitimiste, grand admirateur de Joseph de Maistre (c'est lui qui prête «tous les ouvrages» de celui-ci aux deux héros). Le déséquilibre que l'on a constaté dans le cas de Jeufroy, entre l'abondance des notes de lecture et leur mise en œuvre très réduite, concerne aussi ce personnage emblématique du parti de l'Ordre et de la politique réactionnaire. En effet, dans le chapitre de la religion, les éléments constitutifs de son discours traditionaliste et autoritaire - nostalgie de l'ancien régime, haine de la révolution et de la science, désir du «bras de fer », etc., où l'on est bien tenté de chercher des traces des notes prises sur l'auteur des Soirées - sont en réalité tirés d'autres écrivains catholiques, tels qu'Auguste Nicolas, le père Joseph Félix, Louis-Gaston de Ségur ${ }^{63}$. Il faudrait une étude consacrée à l'utilisation des notes prises sur ces auteurs alimentant les « raisons politiques » évoquées dans le scénario, mais on peut déjà supposer que le romancier a utilisé principalement les sources « secondaires » pour mettre en scène une filiation intransigeante dans le domaine politique.

\section{Conclusion}

Nous avons tenté de mettre en lumière le traitement du documentaire pour la construction d'un personnage qui se définit avant tout par son orientation idéologique. Le personnage du roman n'est plus un type balzacien qui laisse deviner une épaisseur psychologique par des traits extérieurs, mais un être fait de livres dont l'érudition condense des savoirs religieux empruntés à divers ouvrages apologétiques. Le type flaubertien du prêtre nourri des lieux communs incarne aussi le style ecclésiastique dont l'éternelle banalité fascine le romancier ${ }^{64}$. Cette mosaïque de savoirs dissimule pourtant une généalogie des idées, comme nous l'avons vu, dont Joseph de Maistre est l'un des initiateurs et qui reflète l'histoire de l'Église du xixe siècle aux prises avec le monde moderne. Cette historicité que la fiction tend à effacer dans un discours apparemment homogène n'est comprise qu'à travers l'examen des notes de lecture.

\section{NOTES}

1. Voir Jean-Yves Pranchère, L'autorité contre les Lumières: la philosophie de Joseph de Maistre, Genève, Droz, 2004.

2. Stéphanie Dord-Crouslé, «Flaubert et la "religion moderne" ». À partir du dossier "Religion » de Bouvard et Pécuchet ", Revue Flaubert, n 4, 2004 (http://flaubert.univ-rouen.fr), p. 3.

3. S. Dord-Crouslé, «Entre notes de lecture et fragments préparés pour le second volume : les transferts de citations à l'épreuve du dossier "Religion" ", Éditer le chantier documentaire de Bouvard et Pécuchet. Explorations critique et premières réalisations numériques, Andrea Lippolis editore, 2010, p. 81-95. 
4. Anne Herschberg-Pierrot, "La mise en texte des savoirs dans Bouvard et Pécuchet », La mise en texte des savoirs, textes réunis par Kazuhiro Matsuzawa et Gisèle Séginger, Strasbourg, Presses universitaires de Strasbourg, 2010, p. 321-331.

5. Un grand nombre d'éléments sont pourtant repérés par Alberto Cento et Lea Caminiti Pennarola, Commentaire de Bouvard et Pécuchet, Napoli, Liguori, 1973.

6. Scénarios de Bouvard et Pécuchet, ms gg 10, F³0 (Euvres complètes de Gustave Flaubert, Club de l'Honnête Homme, t. 6, 1972, p. 696).

7. Lettre à George Sand du 3 février 1873 (Corr., IV, p. 642). Les références à la correspondance de Flaubert renvoient à la «Bibliothèque de la Pléiade ", Gallimard, édition de Jean Bruneau (I-IV, 1971-1998), et de Jean Bruneau et Yvan Leclerc (V, 2007).

8. Ces notes de lecture sont transcrites dans notre thèse, Taro Nakajima, Les Figures religieuses dans l'œuvre de Gustave Flaubert, thèse de doctorat présentée et soutenue à l'Université Paris-Est (sous la direction de Gisèle Séginger), 2009. Ces transcriptions sont disponibles sur le site du projet de Bouvard et Pécuchet dirigé par Stéphanie Dord-Crouslé (http://dossiers-flaubert.ishlyon.cnrs.fr)

/

9. À Edma Roger des Genettes, 7/9/1873, Corr., IV, p. 712. Sur les rapports entre Flaubert et Maistre, voir Stéphanie Dord-Crouslé, «La face cachée de l'“impartialité" flaubertienne : le cas embarrassant de Joseph de Maistre ", La Bibliothèque de Flaubert. Inventaires et critiques, Rouen, Publications de l'Université de Rouen, 2001, p. 323-336.

10. Bouvard et Pécuchet, édition de Stéphanie Dord-Crouslé, Flammarion, «GF », 2008 [BP], p. 328.

11. g226 (6) fo $246 \mathrm{v}^{\circ}$ et repris au fo323 (notes de notes sur le péché originel). Flaubert a lu ou plutôt relu cet ouvrage en octobre 1879 : « je lis des choses stupides ou plutôt stupidifiantes : les brochures religieuses de Mgr de Ségur, les élucubrations du p[ère] Huguet, jésuite, Baguenault de Puchesse, etc. ! et cet excellent M. Nicolas [...] La Religion moderne est quelque chose d'ineffable, décidément " (à Edma Roger des Genettes, du 8 octobre 1879, Corr., V, p. 720). L'examen des décalages entre les notes de lecture et les notes préparées pour le second volume permet de supposer des relectures successives de l'ouvrage de Baguenault (voir S. Dord-Crouslé, «Entre notes de lectures et fragments préparés pour le second volume: les transferts de citations à l'épreuve du dossier "Religion" ", op. cit., p. 89-90).

12. Le Catholicisme présenté dans l'ensemble de ses preuves, Gaume frères, 1859, t. II, p. 19-20.

13. Ibid., t. I, p. 66.

14. Antoine Compagnon, "Joseph de Maistre et le péché originel continué », La pensée du paradoxe: approches du romantisme: hommage à Michel Crouzet, Presses de l'Université ParisSorbonne, 2006, p. 61-71.

15. Les Soirées de Saint-Pétersbourg, Euvres suivies d'un Dictionnaire Joseph de Maistre, texte établi, annoté et présenté par Pierre Glaudes, Robert Laffont, « Bouquins », 2007, p. 484 ( $2^{\mathrm{e}}$ entretien).

16. A. Compagnon, op. cit., p. 68-69.

17. Le catholicisme présenté, t. I, p. 10.

18. Ibid., p. 48.

19. Ibid., p. 9 , note.

20. «Le péché originel est un mystère sans doute; cependant, si l'homme vient à l'examiner de près, il se trouve que ce mystère $a$, comme les autres, des côtés plausibles, même pour notre intelligence bornée » (Les Soirées, $2^{\mathrm{e}}$ entretien, p. 485).

21. «Chateaubriand, qui prend parfois à ces penseurs [Maistre et Bonald] certains de leurs accents [...] est beaucoup plus proche en réalité du libéralisme conservateur de Burke et de son pragmatisme politique » (Dictionnaire Joseph de Maistre, op. cit., art. "Chateaubriand » par Pierre Glaudes, p. 1147).

22. «La prise de notes de Flaubert est loin d'être toujours fiable. Malgré sa prétention et son désir d'objectivité, [...] le romancier se laisse emporter par ses passions et comprend mal, ou 
seulement partiellement, ce que l'auteur de l'ouvrage consulté voulait dire. Cette partialité de fait a naturellement d'autant plus de chance de se manifester que le romancier éprouve moins de sympathie pour l'auteur » (S. Dord-Crouslé, « La face cachée de l'“impartialité" flaubertienne : le cas embarrassant de Joseph de Maistre », op. cit. p. 335).

23. Les Soirées, $2^{\mathrm{e}}$ entretien, p. 485 (c'est Maistre qui souligne).

24. A. Compagnon, op. cit., p. 68.

25. Les Soirées, $9^{\mathrm{e}}$ entretien, p. 709.

26. g226 (6), fo $279 \mathrm{v}^{\circ}$. L'édition lue par Flaubert est : Les Soirées de Saint-Pétersbourg, ou Entretiens sur le gouvernement temporel de la Providence, suivis d'un Traité sur les sacrifices, $6^{\mathrm{e}}$ éd., Lyon, J.-B. Pélagaud et $C^{\text {ie }}, 1850,2$ vol.

27. Voir la lettre à Edma Roger des Genettes de janvier 1860 (Corr., III, p. 72) où il défend Voltaire en citant une note de l'Éclaircissement sur les sacrifices (Flaubert écrit « traité des Sacrifices »).

28. «Il s'agit de sang, il s'agit de l'immolation proprement dite; il s'agit d'expliquer comment les hommes de tous les temps et de tous les lieux avaient pu s'accorder à croire qu'il y avait, non pas dans l'offrande des chairs (il faut bien observer ceci), mais dans l'effusion du sang, une vertu expiatrice utile à l'homme » (Éclaircissement sur les sacrifices, CEuvres suivies d'un Dictionnaire Joseph de Maistre, op. cit., p. 825-826).

29. Ibid., p. 812 (c'est Maistre qui souligne).

30. «La vitalité du sang, ou plutôt l'identité du sang et de la vie [est] posée comme un fait dont l'antiquité ne doutait nullement, et qui a été renouvelé de nos jours » (Éclaircissement, p. 812).

31. Ibid., p. 833.

32. Bouvard dénonçant l'inutilité de la rédemption car tous les hommes sont damnés «malgré le sacrifice de Notre-Seigneur ", Jeufroy dit: "Mais quotidiennement, il le renouvelle dans l'Eucharistie. » (347) À propos du sacrifice de Jésus renouvelé dans la communion, Maistre écrit : «Il est entré dans les incompréhensibles desseins de l'amour tout-puissant de perpétuer jusqu'à la fin du monde [...], ce même sacrifice, matériellement offert une seule fois par le salut du genre humain. [...] Cette chair divinisée et perpétuellement immolée est présentée à l'homme sous la forme extérieure de sa nourriture privilégiée » (Éclaircissement, p. 838).

33. Le Second volume de Bouvard et Pécuchet, le projet du "Sottisier ", édition d'Alberto Cento et Lea Caminiti Pennarola, Naples, Liguori, 1981, XXXI.

34. Dictionnaire de Joseph de Maistre, op. cit., art. « Tradition » par Jean-Yves Pranchère, p. 1301.

35. Les Soirées, $4^{\mathrm{e}}$ entretien, p. 568.

36. Ibid., $7^{\mathrm{e}}$ entretien, p. 678.

37. À l'ermite affirmant que le martyre prouve la vérité de la doctrine, Hilarion demande: «Comment peut-il en prouver l'excellence, puisqu'il témoigne également pour l'erreur?» ( $\mathrm{L} a$ Tentation de saint Antoine (1874), Gallimard, « Folio », 1983, p. 91) Sur les trois versions de ce texte, voir l'étude fondamentale de Gisèle Séginger, Naissance et métamorphoses d'un écrivain, Flaubert et Les Tentations de saint Antoine, Honoré Champion, 1997. Sur la critique religieuse par le Diable, voir aussi notre article, Taro Nakajima, « Transformation des savoirs dans La Tentation de saint Antoine - du voltairianisme au renanisme ", Études de Langue et Littérature Françaises, Société Japonaise de Langue et Littérature Françaises, $n^{\circ}$ 98, mars 2011, p. 119-131 (en Japonais).

38. Histoire de Jésus-Christ d'après la science ou Le Christianisme proclamé, conformément à la Bible, à l'Évangile et aux Pères, par la religion, les lettres, les arts, les monuments de tous les âges et de tous les pays, depuis le premier homme jusqu'à présent, Paris ; Lyon, Libr. de Bauchu, 1866, p. 5.

39. Preuves éclatantes de la Révélation par l'histoire universelle ou Monuments et témoignages païens, juifs et de tous les peuples, confirmateurs de la Bible et du christianisme, Paris, V. Palmé, $4^{\mathrm{e}}$ éd.,1878, Avantpropos, $\mathrm{XV}$.

40. Ibid., p. 8 (« Idée générale de l'ouvrage »).

41. Ibid., p. 267-268.

42. Ibid., p. 271. 
43. Ibid., p. 270 (Friedrich Creuzer, considérées principalement dans leurs formes symboliques et mythologiques (refondu en partie, complété et développé par Joseph-Daniel Guigniaut, Paris, Teuttel et Würtz, t. I-2, 1825, p. 959. Il s'agit des notes du livre troisième écrites par Guigniaut.

44. Dans les notes prises sur les Preuves éclatantes, on trouve ces fragments avec la vedette «Les Sibylles » en marge : «Le texte de ces vers a pu être interpolé, nous ne le contestons pas. Mais celui qui voudrait les révoquer en doute \& en nier le fond serait aisément confondu par des monuments historiques importants! » (fo202). Flaubert attrape un des rares moments où l'auteur cherche à pallier la faiblesse de sa méthode historique. La vedette accompagnée d'une croix parenthétique montre que ces fragments sont destinés au second volume (classés dans la catégorie « Histoire » dans l'édition de Cento et Caminiti, op. cit. p. 300).

45. Voir Jean-Claude Drouin, «Un légitimiste mystique du $\mathrm{XIX}^{\mathrm{e}}$ siècle: Adrien Péladan 1815-1890 ", Les Péladan, dossier conçu et dirigé par Jean-Pierre Laurant et Victor Nguyen, Lausanne ; Paris, l'Âge d'homme, 1990, p. 14. Cet article de Laurentie paru dans L'Union (janvier 1866) est reproduit au début de l'Histoire de Jésus-Christ d'après la science.

46. Baguenault avoue volontiers ses emprunts faits aux grands devanciers : « Nous leur devons la plupart des pensées de ce travail, nous avons souvent emprunté jusqu'à leurs expressions, croyant qu'ici le seul but, comme le seul mérite, doit être, en dehors de toute revendication personnelle, de donner le plus de relief et d'éclat possible à la vérité » (Le catholicisme présenté, t. I, p. 9 , note).

47. Le comte affirme au septième entretien des Soirées : « Les voyageurs modernes ont trouvé en Amérique les vestales, le feu nouveau, la circoncision, le baptême, la confession, et enfin la présence réelle sous les espèces du pain et du vin " (Les Soirées, p. 679).

48. Pour Maistre, « seule l'infaillibilité du chef de l'Église peut garantir l'unité de la foi en même temps que l'infaillibilité de l'Église » (Dictionnaire de Joseph de Maistre, op. cit., "Infaillibilité ", p. 1196).

49. Le bref est «de caractère privé, sur des matières de moindre importance que celles dont traite la bulle » (Le Robert). Le présent bref est daté du 8 février 1867 et signé par le cardinal Clarelli Paracciani.

50. Preuves éclatantes, V.

51. «La commune, immolant les ôtages, a été le produit du scepticisme et de l'impiété de notre âge" (Preuves éclatantes, p.7), passage pris en note par Flaubert: "Libres penseurs = Communards » (f॰202), qu'il destine au second volume avec une croix parenthétique.

52. «Enfin, ajouta-t-il [...] si l'Église a condamné les spectacles, c'est qu'elle avait raison; il faut nous soumettre à ses décrets " (Madame Bovary, Librairie Générale Française, Le Livre de Poche, 1999, p. 336).

53. En citant une lettre de Flaubert («Tant qu'on n'aura pas détruit le respect pour ce qui est imprimé, on n'aura rien fait!»), A. Herschberg-Pierrot écrit: "Le respect de l'imprimé, la soumission à la chose écrite, c'est celui-là qui prend Bouvard et Pécuchet au piège de la littéralité des signes et de l'autorité des livres, au point qu'ils ne peuvent que les copier - adhésion et adhérence - ou bien qu'ils ne lisent que les passages précédemment soulignés par d'autres lecteurs, qui jugent pour eux ce qu'il faut détacher " (Le Dictionnaire des idées reçues de Flaubert, Presses universitaires de Lille, 1988, p. 33).

54. Le catholicisme présenté, t. I, approbation liminaire non paginée.

55. f० 296 (fragment destiné à la catégorie « Grands Hommes » du second volume).

56. Voir Roger Aubert, Nouvelle Histoire de l'Église 5, sous la direction de L. J. Rogier, Roger Aubert, David Knowles, l'Église dans le monde moderne (1848 à nous jours), Seuil, 1975, p. 49.

57. Ce passage de l'Histoire de Jésus-Christ d'après la science (p. 446) est supprimé dans les Preuves éclatantes.

58. Le traditionalisme de Maistre diffère de celui de Bonald. Voir l'article «Tradition " par JeanYves Pranchère (Dictionnaire de Joseph de Maistre, op.cit., p.1301-1302). Voir aussi 
«Traditionalisme» du Dictionnaire de théologie catholique, Letouzey et Ané, vol. 15, t. 1, 1946, p. 1349 ; Jean-Yves Pranchère, L'autorité contre les Lumières, op. cit., p. 311.

59. Jean-Marie Mayeur, "Catholicisme intransigeant, catholicisme social, démocratie chrétienne », Annales. Économies, Sociétés, Civilisations, $27^{\mathrm{e}}$ année, N. 2, 1972, p. 488 . Voir aussi l'aperçu historique par Laurent Frölich, Les catholiques intransigeants en France, L'Harmattan, 2002, p. 9-14.

60. J.-M. Mayeur, ibid. Bonald et Veuillot, grandes figures de ce courant, ne sont pas dans les notes de lecture pour le neuvième chapitre, tandis que l'on y trouve l'Essai sur l'indifférence en matière de religion, ouvrage le plus représentatif du "premier Lamennais ", c'est-à-dire de sa période maistrienne.

61. Mais il faudrait se rendre compte du contexte dans lequel se situe cette proposition, car «ce qui est rejeté, c'est le progrès et la civilisation moderne tels que les entendent les ennemis de l'Église, c'est-à-dire consistant essentiellement à rejeter l'influence de l'Église dans la société » (Roger Aubert, Le pontificat de Pie IX, Histoire de l'Église depuis les origines jusqu'à nos jours, 21, Bloud et Gay, 1964, p. 255).

62. Baguenault de Puchesse explique le caractère vulgarisateur de son ouvrage qui peut « rendre quelques services à un grand nombre de lecteurs, aussi bien aux hommes occupés qui ont peu de temps à consacrer à l'étude, pourtant si importante, de la Religion, qu'aux hommes de loisir, qu'effrayent et rebutent bien vite les longues recherches intellectuelles. » (Le catholicisme présenté ,t. I, p. 10-11) Quant à Péladan, il est « illustrateur de l'illuminisme en tant qu'historien ou plutôt vulgarisateur des prophéties » (Jean-Claude Drouin, op. cit., p. 16).

63. Flaubert a pris de nombreuses notes sur les opuscules de Mgr de Ségur pour le neuvième chapitre et le second volume de Bouvard (voir leurs transcriptions dans notre thèse). En prenant comme exemple deux ouvrages lus par le romancier (Mgr de Ségur, Réponses courtes et familières..., l'abbé Maunoury, Soirées d'automne), Stéphanie Dord-Crouslé interroge l'émergence d'un genre discursif original, la « causerie apologétique » qui se trouve au croisement entre discours édifiant et style journalistique, entre conférence et récit («Prouver sans s'interdire de plaire: permanence et renouvellement du discours apologétique catholique sous le Second Empire ", Les Religions du xixe siècle, actes du IV congrès de la SERD (novembre 2009), mis en ligne 2011, Société des études romantiques et dix-neuviémistes (http://etudes-romantiques.ish-lyon.cnrs.fr/ religions.html).

64. «Aujourd'hui l'encyclique du pape est bien belle, [...] Quel bon vieux style poncif que le style ecclésiastique! Ce serait, du reste, une étude à faire que celle des styles professionnels ! (lettre à Louis Bouilhet du 5 octobre 1860, Corr., III, p. 118).

\section{INDEX}

Mots-clés : note de lecture, Maistre (Joseph de), religion

\section{AUTEUR}

TARO NAKAJIMA 